\title{
碳纳米管改性海泡石多孔陶瓷及其高效油水分离性能研究
}

\author{
董龙浩 ${ }^{1}$, 张海军 ${ }^{1}$, 张 俊 ${ }^{1}$, 吴文浩 ${ }^{1}$, 贾全利 ${ }^{2}$
}

(1. 武汉科技大学 省部共建耐火材料与冶金国家重点实验室, 武汉 430081；2. 郑州大学 河南省高温功能材料重 点实验室, 郑州 450052)

摘 要: 为了有效地从油/水混合液体中回收油, 本工作以纤维状海泡石为原料, 硝酸镍为催化剂前驱体, 聚乙烯粉 体为造孔剂和碳源, 采用冷冻干燥结合催化裂解法制备了超疏水/超亲油碳纳米管(CNTs)改性海泡石多孔陶瓷, 研 究了固含量和催化热解温度对改性多孔陶瓷形貌的影响, 并表征了其在 $\mathrm{pH}=1$ 的强酸、 $\mathrm{pH}=14$ 的强碱、 $373 \mathrm{~K}$ 高温 和 $77 \mathrm{~K}$ 低温等极端环境中的表面润湿性能及水油分离性能。结果表明: 催化剂前驱体溶液浓度为 $0.5 \mathrm{~mol} / \mathrm{L}$ 、海泡 石的固含量为 $15 \mathrm{wt} \%$ 、催化热解温度为 $973 \mathrm{~K}$ 且保温时间为 $2 \mathrm{~h}$ 时所制备的 CNTs 改性多孔陶瓷具有最好的超疏水 /超亲油性能, 其对柴油、白油、植物油和真空厡油的最高吸附量分别是其自身质量的 15.7、20.8、23 和 25 倍; 其 连续油水分离时油通量高达 $250 \mathrm{~kg} \cdot \mathrm{s}^{-1} \cdot \mathrm{m}^{-2}$, 且在 $5 \mathrm{~h}$ 内分离效率及选择性不发生明显降低。

关 键 词: 海泡石多孔陶瓷; 冷冻干燥; 碳纳米管; 超疏水/超亲油; 油水分离

中图分类号: TQ174 文献标识码: A

\section{Carbon Nanotube Modified Sepiolite Porous Ceramics for High-efficient Oil/Water Separation}

\author{
DONG Longhao ${ }^{1}$, ZHANG Haijun ${ }^{1}$, ZHANG Jun ${ }^{1}$, WU Wenhao ${ }^{1}$, JIA Quanli ${ }^{2}$
}

(1. The State Key Laboratory of Refractories and Metallurgy, Wuhan University of Science and Technology, Wuhan 430081, China; 2. Henan Key Laboratory of High Temperature Functional Ceramics, Zhengzhou University, Zhengzhou 450052, China)

\begin{abstract}
To efficiently recovery oil from oil/water mixed liquid, fibrous sepiolite, polyethylene powder and nickel nitrate was respectively used as raw mateials, carbon source and pore-forming agents, and catalyst precursor to prepare carbon nanotubes (CNTs) modified sepiolite porous ceramics by freeze-drying/catalytic pyrolysis method, and the effects of solid content and pyrolysing temperature on the morphology of the modified porous ceramics were studied. The water contact angle and oil-water separation performance in severly environment such as acid $(\mathrm{pH}=1)$, alkali $(\mathrm{pH}=14)$, and high $(373 \mathrm{~K})$ and low temperature $(77 \mathrm{~K})$ were also characterized. The results showed that when the concentration of catalyst precursor solution was $0.5 \mathrm{~mol} / \mathrm{L}$, the solid content of sepiolite was $15 \mathrm{wt} \%$, the catalytic pyrolysis temperature was $973 \mathrm{~K}$, and the soaking time was $2 \mathrm{~h}$, the as-prepared CNTs modified porous ceramics possessed superhydrophobic/superlipophilic nature, and their maximum adsorption capacity for diesel, paraffin oil, vegetable oil, and vacuum pump oil is respectively 15.7, 20.8, 23 and 25 times of their own weight. The oil flux during the
\end{abstract}

收稿日期: 2019-07-24; 收到修改稿日期：2019-09-24

基金项目: 国家自然科学基金(51872210，51672194); 湖北省教育厅高等学校优秀中青年科技创新团队计划(T201602); 湖 北自然科学基金创新群体项目(2017CFA004)

National Natural Science Foundation of China (51872210, 51672194); Program for Innovative Teams of Outstanding Young and Middle-aged Researchers in the Higher Education Institutions of Hubei Province (T201602); Key Program of Natural Science Foundation of Hubei Province (2017CFA004)

作者简介：董龙浩(1992-)，男，硕士研究生. E-mail: donglonghao1125@163.com DONG Hailong (1992-), male, Master candidate. E-mail: donglonghao1125@163.com

通讯作者: 张海军, 教授. E-mail: zhanghaijun@wust.edu.cn ZHANG Haijun, professor. E-mail: zhanghaijun@wust.edu.cn 
continuous separation process is up to $250 \mathrm{~kg} \cdot \mathrm{s}^{-1} \cdot \mathrm{m}^{-2}$, and the separation efficiency and selectivity can be maintained without significant decreasing within $5 \mathrm{~h}$.

Key words: sepiolite porous ceramics; freeze drying; carbon nanotubes; superhydrophobic/superlipophilic; oil/water separation

近年来, 频发的石油泄漏以及工业和生活含油 废水的随意排放对人类生态系统和健康产生巨大威 胁。对油污及有机污染物的收集和处理引起了研究 人员的广泛关注 ${ }^{[1]}$ 。基于仿生学原理，具有荷叶结构 的自清洁表面、蚊子眼睛结构的防雾涂层以及甲虫 翅膀结构的超疏水表面等被成功制备, 并在油水分 离领域显示出较大的应用潜力 ${ }^{[2]}$ 。其中, 三维多孔材 料由于其高孔隙率及大比表面积, 具有远高于一维 与二维材料的水油分离效率。但它们通常是由海绵 ${ }^{[3]}$ 、 石墨烯 ${ }^{[4-5]}$ 和碳纳米管 ${ }^{[6-7]}$ 等组装而成，存在机械强 度低及耐酸碱腐蚀性差的问题，在一定程度上限制 了其在极端环境下的应用。因此，开发和制备具有 高油水分离效率、较高的力学强度及良好化学稳定 性的三维多孔材料具有重要意义。

多孔陶瓷具有显气孔隙率高、比表面积大、耐 磨性高及化学稳定性好等特点 ${ }^{[8-9]}$, 是理想的过滤 吸附材料 ${ }^{[10]}$ 。其制备方法主要有: 浸渍法 ${ }^{[11]}$ 、添加 造孔剂法 ${ }^{[12]}$ 、发泡法 ${ }^{[13]}$ 及冷冻干燥法 ${ }^{[14]}$ 等。其中冷 冻干燥法具有操作简单、适用范围广、环保可靠等 优点, 并且能够制备具有特殊定向孔道结构的材料, 已被广泛用于制备氧化铝 ${ }^{[15]}$ 、硼化锆一碳化硅 ${ }^{[16]}$ 、 氮化硅 ${ }^{[17]}$ 及氧化铁 ${ }^{[18]}$ 等多孔陶瓷材料。但传统的多 孔陶瓷往往既亲水又亲油, 因此, 需对其进行表面 改性，使其疏水亲油、具有良好的油水分离性能。

本课题组前期的研究结果表明 ${ }^{[19]}$, 经石墨烯/ 碳纳米带改性后, 亲油疏水的硅藻土多孔陶瓷具有 良好的的油水分离能力和较高的力学强度。但是该 工艺需要先采用发泡法制备硅藻土多孔陶瓷，再用 石墨烯进行表面改性，最后用催化裂解法在多孔陶 瓷表面原位化学沉积碳纳米带，制备工艺复杂; 同 时, 所制备样品的油水分离能力还有待进一步优化。

纤维状海泡石具有成本低、来源广、储量大、 安全环保、比表面积大及吸附性能良好等特点, 在 吸附和净化领域应用广泛 ${ }^{[20-23]}$ 。海泡石优良的吸附 性能取决于其独特的结构: 1) 硅氧四面体和镁氧八 面体形成的层链状结构使其具有了巨大的理论比表 面积; 2) 纤维状海泡石内部分布着分子尺寸的微孔 通道，这些贯通的通道赋予其较大的理论孔容积。 海泡石的这些特性是其在物理吸附方面得以广泛应 用的关键 ${ }^{[24-26]}$ 。
本工作以纤维状海泡石为原料, 硝酸镍为催化 剂前驱体, 聚乙烯(PE)粉体为造孔剂和碳源, 采用 冷冻干燥结合催化裂解法一步制备了碳纳米管 (CNTs) 改性海泡石多孔陶瓷, 并研究了其在 $\mathrm{pH}=1$ 的强酸、 $\mathrm{pH}=14$ 的强碱、 $373 \mathrm{~K}$ 高温和 $77 \mathrm{~K}$ 低温等 环境中的表面润湿性能及水油分离性能。

\section{1 实验方法}

\section{1 材料制备}

实验原料主要有纤维状海泡石 $\left(\mathrm{Mg}_{8} \mathrm{Si}_{12} \mathrm{O}_{30}(\mathrm{OH})_{4}\left(\mathrm{OH}_{2}\right)_{4} \cdot 8 \mathrm{H}_{2} \mathrm{O}\right)($ 工业级 $) 、$ 聚乙烯粉 $\left(\left(\mathrm{C}_{2} \mathrm{H}_{4}\right) n, \mathrm{PE}, 97 \%\right)$ 、硝酸镍 $\left(\mathrm{Ni}\left(\mathrm{NO}_{3}\right)_{2} \cdot 9 \mathrm{H}_{2} \mathrm{O}\right)$ (分析 纯)、羧甲基纤维素钠 $\left(\mathrm{C}_{8} \mathrm{H}_{16} \mathrm{NaO}_{8}, \mathrm{CMC}\right.$, 分析纯)和 去离子水。

为了制备结构均匀的海泡石多孔陶瓷，需要对 原料纤维状海泡石进行球磨处理来提高其分散度, 球磨前, 纤维状海泡石团聚成束, 其长度为 $1 \sim 2 \mathrm{~cm}$, 经球磨后所制得的纤维直径为 $5 \sim 10 \mu \mathrm{m}$, 长度为 100 200 $\mu \mathrm{m}$ 。首先配制 $1 \mathrm{wt} \%$ 的羧甲基纤维素钠溶 液, 并加入一定量的硝酸镍, 配置成浓度为 $0.5 \mathrm{~mol} / \mathrm{L}$ 的催化剂前驱体溶液。随后将 $10 \mathrm{wt} \%$ 的聚乙烯粉加 入到硝酸镍溶液中, 充分的磁力搅拌后, 使得催化 剂前驱体均匀地吸附在聚乙烯粉体颗粒表面; 再将 球磨 $2 \mathrm{~h}$ 后的纤维状海泡石按照 $10 \mathrm{wt} \% \sim 20 \mathrm{wt} \%$ 的 固含量加入到上述溶液中经摚拌制得浆料。将浆料 注入模具中经液氮冷冻成型后, 在冷冻干燥机中于 $233 \mathrm{~K}$ 和 $1 \mathrm{~Pa}$ 条件下真空干燥 $48 \mathrm{~h}$ 以去除湿坏中的 水分得到干坯。最后将干坏置于石英管式炉中, 在 $5 \mathrm{vol} \% \mathrm{H}_{2} / \mathrm{Ar}$ 气氛中, 以 $5 \mathrm{~K} / \mathrm{min}$ 的升温速率升温至 923 1023 K, 并保温 $2 \mathrm{~h}$ 。在该过程中，硝酸镍被还 原为镍纳米颗粒，原位催化聚乙烯裂解产生的含碳 气体沉积在多孔陶瓷内部及表面原位生成 $\mathrm{CNTS}^{[27-28]}$, 最终制得 CNTs 改性海泡石多孔陶瓷。

\section{2 材料测试与表征}

采用扫描电子显微镜 (FE-SEM, Nova400NanoSEM, PHILIPS, NETHERLANDS, $15 \mathrm{kV}$ ) 和透射电子显微镜(TEM, JEM-2100UHRSTEM, JEOL, JAPAN, $200 \mathrm{kV}$ )表征试样的显微结构。采用压录仪 (AutoPore IV9510)表征样品气孔率和孔径分布。试 
样表面的润湿性采用接触角测量仪(OCA15EC, Germany)来测量(水滴的体积为 $5 \mu \mathrm{L}$ ), 并分别测量 5 次取其平均值。CNTs 改性多孔陶瓷的碳含量 $(C)$ 、 吸附量 $(Q)$ 、连续油水分离时油通量 $(v)$ 及分离选择性 (w)分别通过公式(1)、(2)、(3)和(4)进行计算:

$$
C=\frac{M_{1}-M_{2}}{M_{1}} \times 100 \%
$$

其中, $M_{1}(\mathrm{~g})$ 代表 CNTs 改性多孔陶瓷的质量, $M_{2}(\mathrm{~g})$ 代表空气气氛下经 $1073 \mathrm{~K}$ 热处理后 CNTs 改性海泡 石多孔陶瓷的质量。

$$
Q=\frac{M_{2}-M_{1}}{M_{1}} \times 100 \%
$$

其中, $M_{1}(\mathrm{~g})$ 代表 CNTs 改性海泡石多孔陶瓷的原始 重量, $M_{2}(\mathrm{~g})$ 代表 CNTs 改性海泡石多孔陶瓷浸入测 试液体中并沥去多余液滴后的重量。

$$
v=\frac{M}{T \times S}
$$

其中, $M(\mathrm{~g})$ 代表 $\mathrm{CNTs}$ 改性海泡石多孔陶瓷分离油 的重量, $T(\mathrm{~s})$ 代表 CNTs 改性海泡石多孔陶瓷水油分 离所用时间, $S\left(\mathrm{~m}^{2}\right)$ 代表在水油分离实验中 CNTs 改 性海泡石多孔陶瓷的有效表面积(因为下表面始终 处于水中不参与油水分离过程，上表面几乎都被密 封胶覆盖, 所以有效表面积选取多孔陶瓷的四个侧 面加以计算)。

$$
w=\frac{V_{1}}{V_{2}} \times 100 \%
$$

其中, $V_{1}$ 为在 $\mathrm{CNTs}$ 改性海泡石多孔陶瓷水油分离 过程中分离的油的体积 $(\mathrm{mL}), V_{2}$ 代表在水油分离过 程中分离的液体总体积 $(\mathrm{mL})$ 。

\section{2 结果与讨论}

\subsection{CNTs 改性前后海泡石多孔陶瓷的形貌}

图 1 为采用冷冻干燥法制备的 CNTs 改性前海 泡石多孔陶瓷及改性后 CNTs 改性海泡石多孔陶瓷 的 SEM 照片。从图中可知: 多孔陶瓷的干坏中主要 存在着单一分散的长海泡石纤维, 图 1(a)中的定向 孔道是冷冻干燥工艺留下的, 而图 1(b)中黑色圆圈 标示的窗口气孔是由海泡石纤维搭接形成的。改性 后, CNTs 改性海泡石多孔陶瓷不仅存在着 PE 原位 裂解之后留下的数百微米的大孔(图 1(c)), 同时还 保留了冷冻干燥工艺所特有的定向孔道结构(图 1(c))。 并且改性后多孔陶瓷层在定向孔道的孔壁上和窗 口型小气孔中原位生成了大量碳纳米管。大量交 错纵横的碳纳米管在样品表面及内部产生相对较
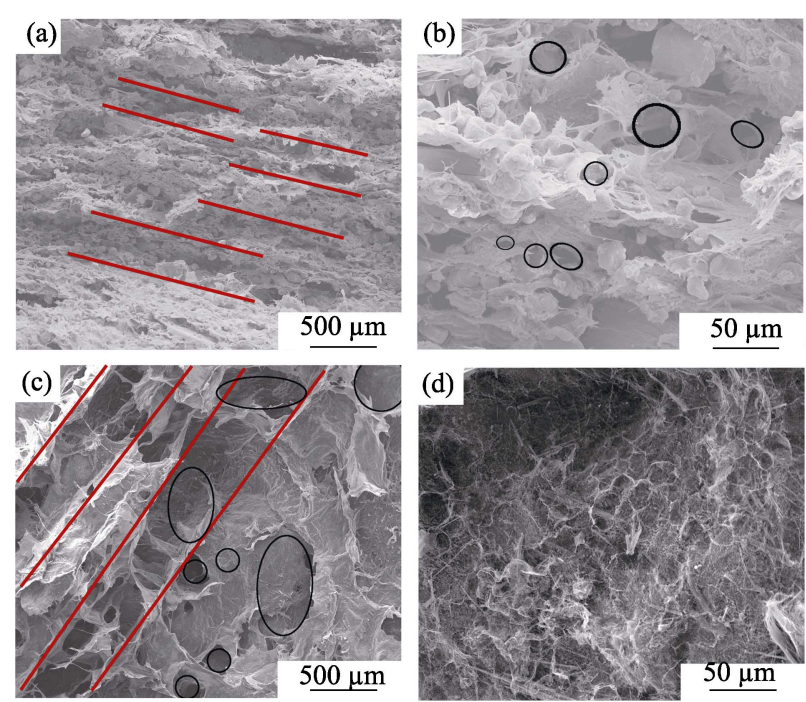

图 1 海泡石多孔陶瓷的 SEM 照片

Fig. 1 SEM micrographs of the as-prepand sepiolite porous ceramics

(a-b) Before CNTs modification; (c-d) Modified with CNTs

高的粗粘度, 有助于样品疏水性能的提高。同时, 碳 纳米管相互交织在一起也可以在一定程度上提高样 品的力学性能(图 1(d))。

\section{2 固含量对 CNTs 改性海泡石多孔陶瓷形貌 的影响}

图 2 为不同海泡石固含量 $(10 \mathrm{wt} \%$ 20wt\%)时, 经 $973 \mathrm{~K} / 2 \mathrm{~h}$ 原位催化裂解后制得 CNTs 改性海泡石 多孔陶瓷的 SEM 照片。当固含量为 $10 \mathrm{wt} \%$ 时(图 2(a)), 产物中存在着少量长度仅有数百纳米且直径约为 $50 \sim 100 \mathrm{~nm}$ 的的短簇状 CNTs; 当固含量增加至 $15 \mathrm{wt} \%$ 时 (图 2(b)), 产物中存在大量长度可达数百 微米且直径为 $30 \sim 50 \mathrm{~nm}$ 的 CNTs; 当固含量增至 $20 \mathrm{wt} \%$ (图 2(c)), CNTs 的长度变短, 仅有数百纳米, 其直径为 30 40 nm, 呈短簇状。除了原位产生的 CNTs 形貌外, 样品中碳的含量(包括 CNTs 及无定形碳) 对改性多孔陶瓷的润湿性影响较大。根据氧化失重 实验结果可知(图 2(d)): 固含量为 $10 \mathrm{wt} \% 、 15 \mathrm{wt} \%$ 和 $20 \mathrm{wt} \%$ 时, 样品的碳含量呈现先增加后减小的趋势, 其值分别为 $2.7 \mathrm{wt} \% 、 20.5 \mathrm{wt} \%$ 和 $10.0 \mathrm{wt} \%$ 。

水润湿角的测试结果表明: 三个试样的水润湿 角分别为 $97^{\circ} 、 140^{\circ}$ 和 $135^{\circ}$ (图 3), 所制备的 CNTs 改性海泡石多孔陶瓷的疏水程度与样品中碳(主要 为 $\mathrm{CNTs}$ )含量关系密切。实验条件下的最佳固含量 为 $15 \mathrm{wt} \%$ 。

\section{3 裂解温度对 CNTs 改性海泡石多孔陶瓷形 貌的影响}

图 4 为在不同温度时所制备固含量为 $15 \mathrm{wt} \%$ 的 

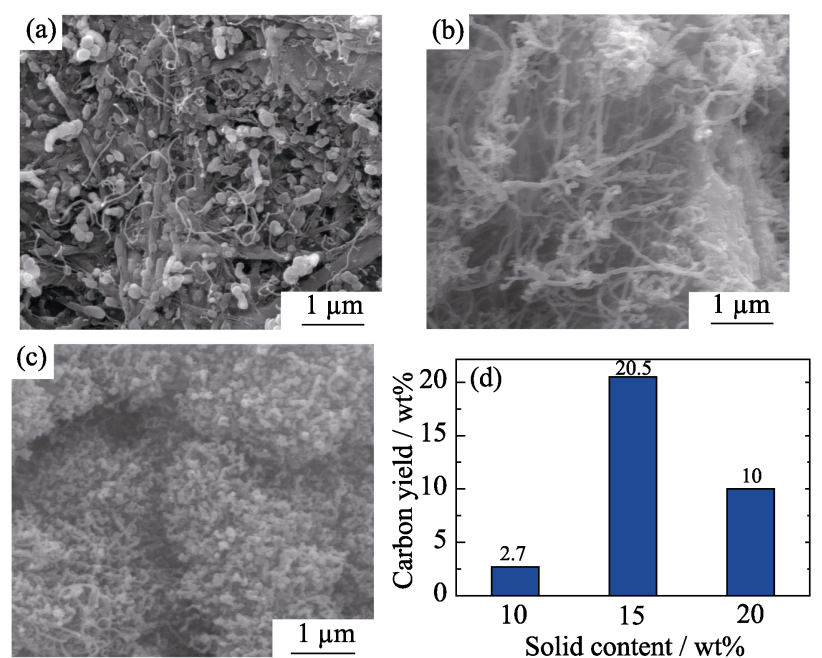

图 2 不同固含量的 CNTs 改性海泡石多孔陶瓷的 SEM 照片 和碳含量变化

Fig. 2 SEM micrographs and carbon content change of CNTs modified sepiolite porous ceramics with various solid contents (a) $10 \mathrm{wt} \%$; (b) $15 \mathrm{wt} \%$; (c) $20 \mathrm{wt} \%$; (d) Carbon content change
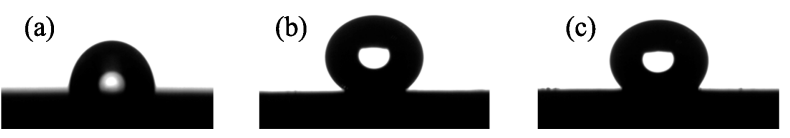

图 3 不同固含量的 CNTs 改性海泡石多孔陶瓷的表面水润 湿角

Fig. 3 The static water contact angle of CNTs modified sepiolite porous ceramics with various solid contents (a) $10 \mathrm{wt} \%$; (b) $15 \mathrm{wt} \%$; (c) $20 \mathrm{wt} \%$

CNTs 改性海泡石多孔陶瓷的 SEM 照片(催化剂前 驱体硝酸镍溶液浓度为 $0.5 \mathrm{~mol} / \mathrm{L}$ )。由图可知: 1) 当 催化裂解温度为 $923 \mathrm{~K}$ 时, 产物中有大量直径约为 30 50 nm 且长度可达几十微米的碳纳米管生成(图 4(a)); 2)当催化裂解温度升高至 $973 \mathrm{~K}$ 时, CNTs 的直径和 长度无明显变化, 但碳纳米管的生成量有所增加(图 4(b))；3）当催化裂解温度为 $1023 \mathrm{~K}$ 时，碳纳米管的 生成量和长度均无明显变化(图 4(c))。不同催化裂解 温度下 CNTs 改性海泡石多孔陶瓷中的碳含量
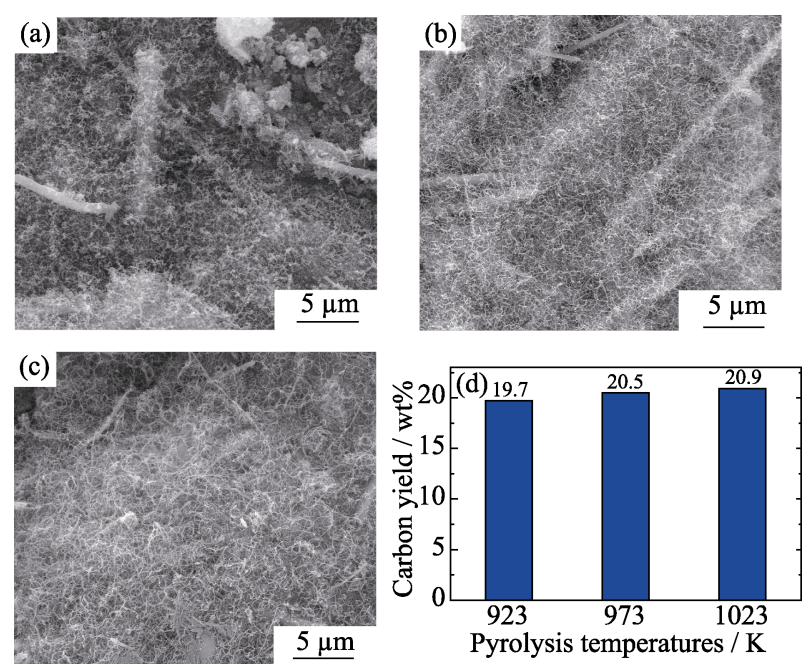

图 4 不同催化裂解温度时 CNTs 改性海泡石多孔陶瓷的 $\mathrm{SEM}$ 照片和碳含量变化

Fig. 4 SEM micrographs and carbon content change of CNTs modified sepiolite porous ceramics prepared at various pyrolysis temperatures

(a) $923 \mathrm{~K}$; (b) $973 \mathrm{~K}$; (c) $1023 \mathrm{~K}$; (d) Carbon content change

分别为 $19.7 \mathrm{wt} \% 、 20.5 \mathrm{wt} \%$ 和 $20.9 \mathrm{wt} \%$ (图 4(d))。由 于在 $973 \mathrm{~K}$ 时 CNTs 的生成量最多, 本研究选择此温 度点为制备 CNTs 改性海泡石多孔陶瓷的催化裂解 温度。

\subsection{CNTs 改性海泡石多孔陶瓷的 TEM 分析}

图 5 为改性海泡石多孔陶瓷中 CNTs 的 TEM 及 HRTEM 照片 $(973 \mathrm{~K} / 2 \mathrm{~h}$ 、固含量 $15 \mathrm{wt} \%$ 、催化剂前 驱体硝酸镍溶液浓度为 $0.5 \mathrm{~mol} / \mathrm{L})$ 。从图可知: 图 5(a) 中的黑色颗粒为原位生成的催化剂 $\mathrm{Ni}$ 纳米颗粒 ${ }^{[36-37]}$, 其粒径为 30 50 $\mathrm{nm}$ 。图 5(b)中的 CNTs 存在明显的 端口, 壁厚约为 $3 \mathrm{~nm}$ 且外径为 $30 \mathrm{~nm}$ (图 5(b)); 由图 5(c)可知, CNTs 的管壁层间距约为 $0.34 \mathrm{~nm}$, 与石墨 的(002)晶面的标准晶面间距一致。

\subsection{CNTs 改性海泡石多孔陶瓷的静态吸附性能}

研究了 $973 \mathrm{~K} / 2 \mathrm{~h}$ 且固含量为 $15 \mathrm{wt} \%$ 的条件下 制备的 CNTs 改性海泡石多孔陶瓷(催化剂前驱体硝
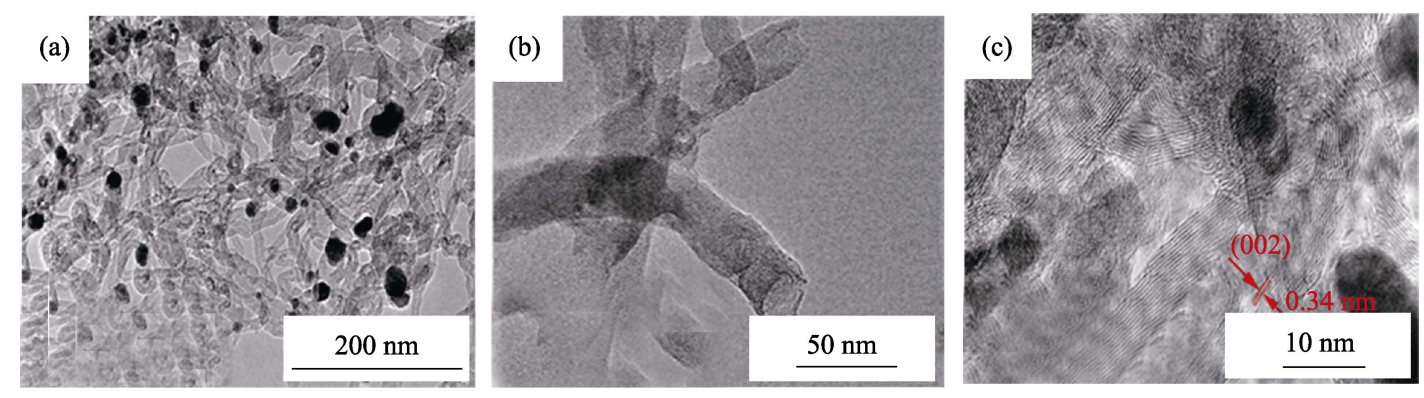

图 $5973 \mathrm{~K} / 2 \mathrm{~h}$ 催化裂解聚乙烯所制备 CNTs 的 TEM 和 HRTEM 照片

Fig. 5 TEM and HRTEM images of CNTs resultant from pyrolysis of polyethylene at $973 \mathrm{~K}$ for $2 \mathrm{~h}$ under the optimal condition (a) Low transmission electron microscopy; (b) Tip of CNTs; (c) HRTEM images of the walls of CNTs 
酸镍溶液浓度为 $0.5 \mathrm{~mol} / \mathrm{L}$ )对不同种类油的吸附性 能。从图 6 可知, CNTs 改性的海泡石多孔陶瓷对柴 油、白油、植物油和真空厡油的吸附量分别为其自 重的 15.7、20.8、23.0 和 25.0 倍。表 1 对比了不同 种类吸附材料对油或有机物的吸附性能，从表中可 知，实验条件下所制备的 CNTs 改性多孔陶瓷具有 优异的油吸附性能; 其吸附量为文献报道数据的 3.5 263 倍。其优异的吸油能力一方面可能是因为多 孔陶瓷表面及内部原位生长的碳纳米管提供较多的 吸附位点; 另一方面，可能与多孔陶瓷的气孔率、孔 结构以及孔径分布有关。

当固含量为 $15 \mathrm{wt} \%$ 时, 样品的气孔率高达 $91.2 \%$ ，其孔径分布如图 7 所示，主要为 50 200 $\mu \mathrm{m}$ 的大孔; 样品超高的孔隙率以及相对较大的孔径均 有利于油的吸附和储存。此外, 冷冻干燥法所特有 的层状定向孔道结构也为各种油类的储存和扩散提 供了空间和渠道。

\subsection{CNTs 改性海泡石多孔陶瓷的连续油水分} 离性能

为了达到可以持续进行水油分离的目的，本工 作将自吸泵和 CNTs 改性海泡石多孔陶瓷相连, 研 究多孔陶瓷的连续油水分离性能。如图 8(a)所示, 首 先在 CNTs 改性海泡石多孔陶瓷单侧打孔，然后将 聚氨酯(PU)管插入孔内, PU 管路和改性多孔陶瓷的 接触位置使用热熔胶进行密封及粘结处理(图 8(b)), 最后将其与自吸泵相连得到连续性油水分离装置。 当进行油水分离时, 水面的浮油可通过毛细管力附
着在 CNTs 改性海泡石多孔陶瓷表面，当自吸葲给 陶瓷提供一个额外的吸力时，陶瓷内部形成一定的 负压，陶瓷表面吸附的油被不断吸入材料内部通过 管道洜入回收装置中(图 8(c))。由于所制备的 CNTs 改性海泡石多孔陶瓷具有疏水亲油的的特性，可以 大大减少水被吸入的概率。在整个水油分离的过程 中，不仅提高了 CNTs 改性海泡石多孔陶瓷的利用 率，同时也缩短了分离时间，节约了能耗。

以水/白油混合液体为对象研究装置的持续工 作能力，实验过程中，多孔陶瓷置于油水界面处， 为了减小实验误差每次收集和回收的时间控制在 $5 \mathrm{~min}$ 以内，每次间隔 $30 \mathrm{~min}$ 测量所回收白油的重量，装 置连续工作 $5 \mathrm{~h}$, 以检测油水分离装置的耐久性。 CNTs 改性海泡石多孔陶瓷的油水分离效率如图 9 所示，其连续油水分离时油通量约为 $250 \mathrm{~kg} \cdot \mathrm{s}^{-1} \cdot \mathrm{m}^{-2}$,

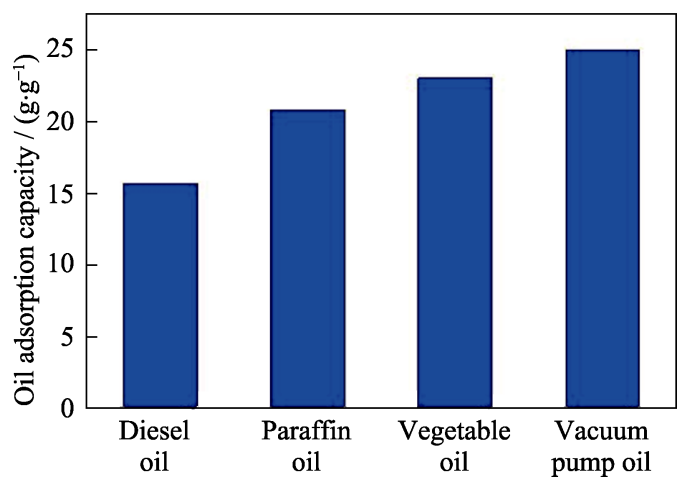

图 6 CNTs 改性海泡石多孔陶瓷对不同油的静态吸附性能 Fig. 6 Static adsorption capacities of CNTs modified sepiolite porous ceramics for different oils

表 1 各种吸附材料吸附量的比较

Table 1 Comparison of adsorption capacity values of various adsorbent materials developed to date

\begin{tabular}{|c|c|c|c|c|}
\hline Adsorbent material & Adsorbate & Modified substance & Adsorption capacity $/\left(\mathrm{g} \cdot \mathrm{g}^{-1}\right)$ & Ref. \\
\hline Sepiolite powders & Motor oil & - & $0.174-0.184$ & [29] \\
\hline Modified silica powders & Motor oil & 1, 7-octadiene & 0.330 & {$[30]$} \\
\hline Diatomite/silicalite- I composite powders & Benzene & Silicalite- I & $0.095-0.246$ & {$[31]$} \\
\hline Modified diatomite porous ceramics & Toluene & Graphene/carbon nanobelts & 1.090 & [19] \\
\hline Modified diatomite porous ceramics & Vacuum pump oil & Graphene/carbon nanobelts & 1.025 & [19] \\
\hline Boron nitride aerogel & Salad oil & - & 5 & {$[32]$} \\
\hline Modified alumina porous ceramics & Hexane & Polydimethylsiloxane & 2 & {$[33]$} \\
\hline Modified rock wool & Diesel oil & Polydimethylsiloxane/silica & 7 & [34] \\
\hline Polymethylsilsesquioxane aerogel & Hexane & - & 6.2 & {$[35]$} \\
\hline Modified sepiolite porous ceramics & Hiesel oil & CNTs & 15.7 & This work \\
\hline Modified sepiolite porous ceramics & Haraffin oil & CNTs & 20.8 & This work \\
\hline Modified sepiolite porous ceramics & Vegetable oil & CNTs & 23 & This work \\
\hline Modified sepiolite porous ceramics & Vacuum pump oil & CNTs & 25 & This work \\
\hline
\end{tabular}




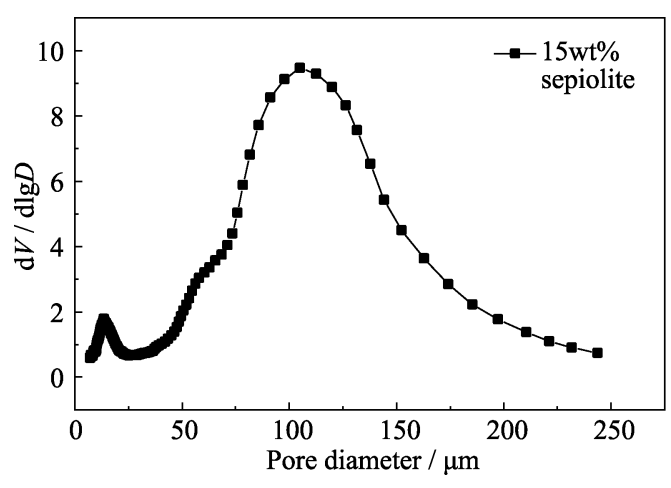

图 7 CNTs 改性多孔陶瓷的孔径分布

Fig. 7 Pore size distribution of CNTs modified sepiolite porous ceramics

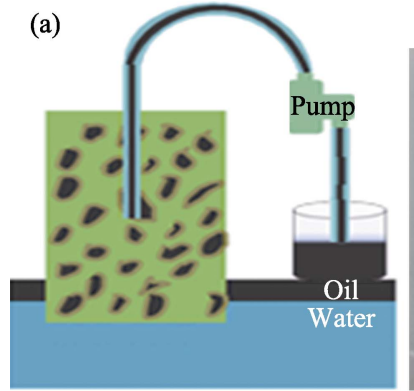

(b)

(c)
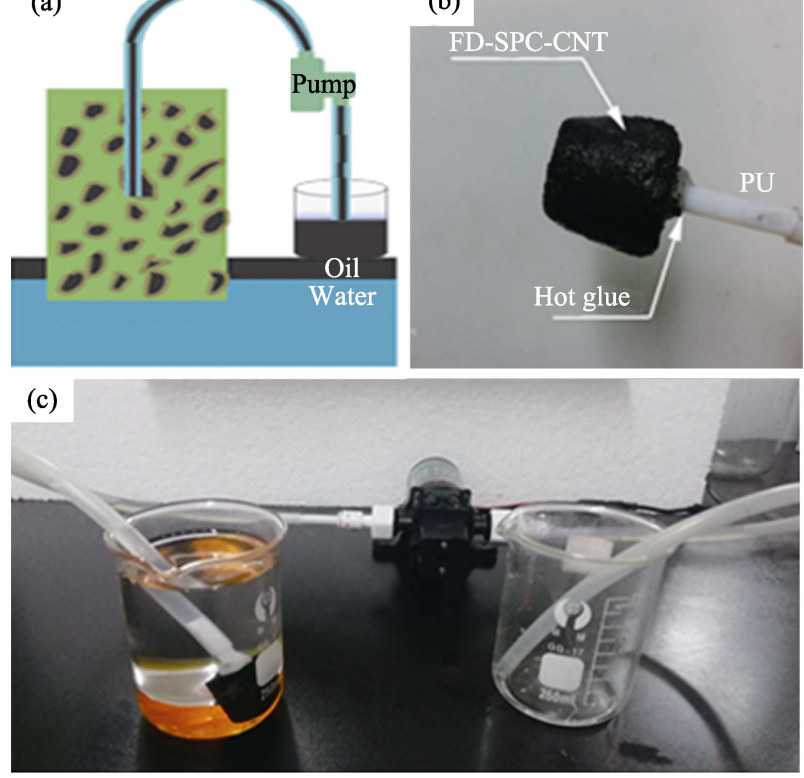

图 8 连续性油水分离装置模拟图(a)和实物图(b c)

Fig. 8 Schematic illustration (a) and images (b-c) of the continuously oil recovery instrument

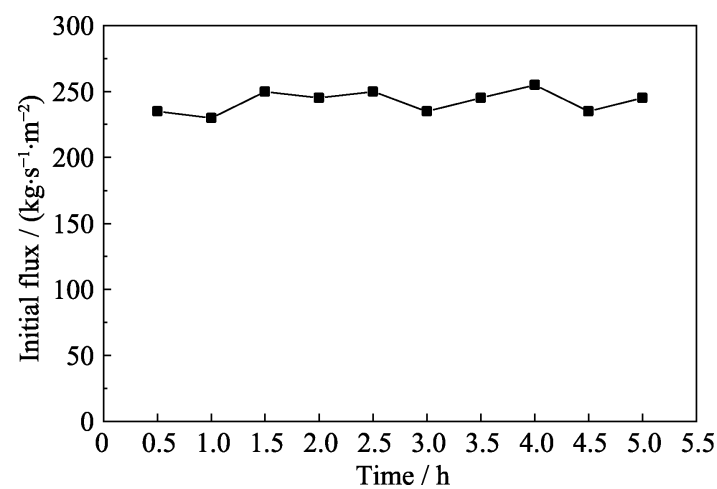

图 $9 \mathrm{CNTs}$ 改性海泡石多孔陶瓷连续工作 $5 \mathrm{~h}$ 的分离油通量

Fig. 9 The oil flux of CNTs modified sepiolite porous ceramics for paraffin oil/water mixture within $5 \mathrm{~h}$

\section{且其分离效率在 $5 \mathrm{~h}$ 内未发生明显变化。}

在油水分离材料的实际应用过程中，常常需要 面对未知极端环境的挑战, 因此对材料的可靠性和 稳定性有着极高的要求。基于此, 本研究分别模拟
和测试了 CNTs 改性海泡石多孔陶瓷在极端环境 $(\mathrm{pH}=1$ 的强酸与 $\mathrm{pH}=13$ 的强碱浸泡, $373 \mathrm{~K}$ 保温 $4 \mathrm{~h}$ 及 $77 \mathrm{~K}$ 保温 $4 \mathrm{~h}$ 等)下的水润湿角及油水分离性能。 首先研究了极端环境下所制备的改性多孔陶瓷的水润 性能: 原始改性多孔陶瓷的水润湿角为 $145^{\circ}$ (图 10(a)), 其经强酸、强碱、高温和低温处理后理后润湿角分 别为 $142^{\circ} 、 143^{\circ} 、 143^{\circ}$ 和 $143^{\circ}$ (图 10(b e))。表明所 制备的 CNTs 改性海泡石多孔陶瓷具有优异的抵抗 极端条件的能力。

进一步表征了极端条件处理前后 CNTs 改性海 泡石多孔陶瓷的连续性水油分离的选择性(图 11)。 从图可知, 极端环境处理前后试样的水油分离选择 性变化不大, 表明制备的 CNTs 改性多孔陶瓷在极 端环境下都具有优秀的油水分离性能。

\subsection{CNTs 改性海泡石多孔陶瓷高效油水分离}

\section{机理分析}

未经碳纳米管改性的海泡石多孔陶瓷既亲油又 亲水, 其水润湿角和油润湿角如图 12 所示, 不具备 油水分离的能力。经碳纳米管改性后，样品的超疏 水性和超亲油性是其油水分离性能优异的两个关键 因素。研究表明: 纳米尺度下, 材料表面总是粗䊁的, 因此材料的润湿界面实际是由液-气和液-固两部分 组成。Wenzel ${ }^{[38]}$ 认为, 材料表面的接触角应满足方程:

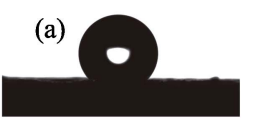

(c)

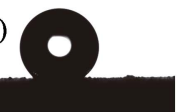

(b)

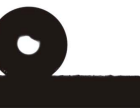

(d) (e)

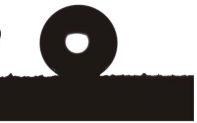

图 $10 \mathrm{CNTs}$ 改性多孔陶瓷在不同环境下的水接触角

Fig. 10 The static water contact angles of CNTs modified sepiolite porous ceramics in various environments

(a) As-synthesized ceramic; (b) $\mathrm{pH}=1$; (c) $\mathrm{pH}=13$; (d) $373 \mathrm{~K}$; (e) $77 \mathrm{~K}$

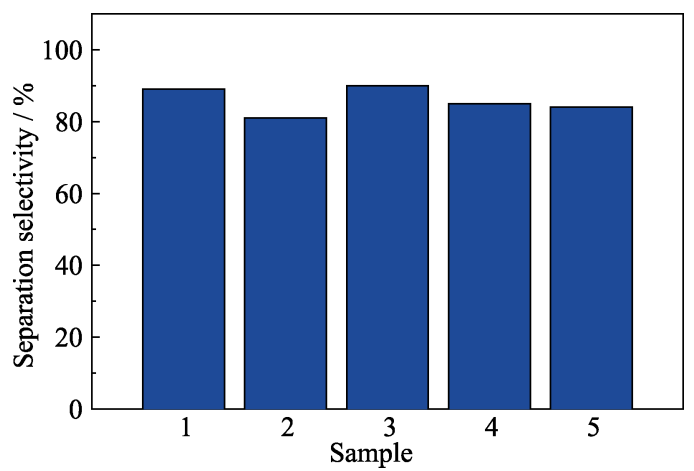

图 11 不同环境下 CNTs 改性多孔陶瓷的油水分离选择性

Fig. 11 Oil/water separation selectiviies of CNTs modified porous ceramics in various environments

(1) As-synthesized ceramic; (2) $\mathrm{pH}=1$; (3) $\mathrm{pH}=13$; (4) Heating at $373 \mathrm{~K}$ for $4 \mathrm{~h}$; (5) Freezing at $77 \mathrm{~K}$ for $4 \mathrm{~h}$ 
(a)

(b)

12 不含碳纳米管的多孔陶瓷的水接触角和油接触角

Fig. 12 Static water/oil contact angle of porous ceramics without CNTs

(a) Water contact angle; (b) Oil contact angle

$$
\cos \theta=\frac{\gamma\left(\gamma_{\mathrm{SA}}-\gamma_{\mathrm{SL}}\right)}{\gamma_{\mathrm{LA}}} \gamma \cos \theta_{1}
$$

其中, $\gamma_{\mathrm{SA}} 、 \gamma_{\mathrm{SL}}$ 和 $\gamma_{\mathrm{LA}}$ 分别代表固/气、固/液和液/ 气的表面张力; $\gamma$ 为粗楉因子且恒大于 $1 ; \theta$ 和

$\theta_{1}$ 分别是表观接触角和本征接触角。由该方程可知, 材料表面粗糙度会影响其润湿性 ${ }^{[39-40]}$ 。改性后的多 孔陶瓷表面及内部孔结构中原位生长着大量碳纳米 管，碳纳米管本身具有疏水的特性; 另一方面，原位 生成的 CNTs 提高了多孔陶瓷表面的粗糙度, 使其 疏水程度提高，更适用于油水分离 ${ }^{[41]}$ 。

疏水亲油的特性以及高的连通孔隙率两方面的 因素使得改性的海泡石多孔陶瓷与自吸沗相连后, 在最大吸力为 $0.5 \mathrm{MPa}$ 的自吸泵驱动下具有优异且 稳定的油水分离性能。

\section{3 结论}

1) 采用冷冻干燥结合催化裂解的方法一步制 备了具有超疏水/超亲油的 CNTs 改性海泡石多孔陶 瓷，制备多孔陶瓷的最佳固含量为 $15 \mathrm{wt} \%$, 最佳裂 解温度为 $973 \mathrm{~K}$ 。改性多孔陶瓷中的碳含量约为 $20.5 \mathrm{wt} \%$, CNTs 的直径约为 $30 \sim 50 \mathrm{~nm}$, 且长度高达 数百微米。

2) 所制备的 CNTs 改性海泡石多孔陶瓷的水润 湿角高达 $145^{\circ}$, 对柴油、白油、植物油和真空洜油 的最高吸附量分别为其自重的 $15.7 、 20.8 、 23.0$ 和 25.0 倍; 并且对白油的连续油水分离时油通量高达 $250 \mathrm{~kg} \cdot \mathrm{s}^{-1} \cdot \mathrm{m}^{-2}$, 油水分离选择性高于 $90 \%$, 且可保 持该分离效率在 $5 \mathrm{~h}$ 内不发生明显降低。

3) 所制备的 CNTs 改性海泡石多孔陶瓷可在强 酸、强碱、高温及低温等极端环境中稳定使用, 并 呈现出超疏水性、高的耐久性及水油分离选择性。

\section{参考文献:}

[1] ZHANG JUN, HAN LEI, ZHANG HAI-JUN, et al. Selective oil/water separation materials. Progress in Chemistry, 2019, 31(1):
$146-155$.

[2] LIU KE-SONG, YAO XI, JIANG LEI. Recent developments in bio-inspired special wettability. Chemical Society Reviews, 2010, 39(8): 3240-3255.

[3] SU CHUN-PING, YANG HAO, SONG SHUANG, et al. A magnetic super-hydrophilic/oleophobic sponge for continuous oil-water separation. Chemical Engineering Journal, 2017, 309: 366-373.

[4] ZHANG LIN, LI HONG-QIANG, LAI XUE-JUN, et al. Thiolated graphene-based superhydrophobic sponges for oil-water separation. Chemical Engineering Journal, 2017, 316: 736-743.

[5] LIU CAN, YANG JIN, TANG YONG-CAI, et al. Versatile fabrication of the magnetic polymer-based graphene foam and applications for oil-water separation. Colloids and Surfaces A, 2015, 468: $10-16$.

[6] SHI ZHUN, ZHANG WEN-BIN, ZHANG FENG, et al. Ultrafast separation of emulsified oil/water mixtures by ultrathin freestanding single-walled carbon nanotube network films. Advanced Materials, 2013, 25(17): 2422-2427.

[7] GUI XU-CHUN, ZENG ZHI-PING, LIN ZHI-QING, et al. Magnetic and highly recyclable macroporous carbon nanotubes for spilled oil sorption and separation. ACS Applied Materials \& Interfaces, 2013, 5(12): 5845-5850.

[8] HAN LEI, DENG XIAN-GONG, LI FA-LIANG, et al. Preparation of high strength porous mullite ceramics via combined foamgelcasting and microwave heating. Ceramics International, 2018, 44(12):14728-14733.

[9] HAN LEI, LI FA-LIANG, HUANG LANG, et al. Preparation of $\mathrm{Si}_{3} \mathrm{~N}_{4}$ porous ceramics via foam-gelcasting and microwave-nitridation method. Ceramics International, 2018, 44(15): 17675-17680.

[10] DENG XIAN-GONG, RAN SONG-LIN, HAN LEI, et al. Foam-gelcasting preparation of high-strength self-reinforced porous mullite ceramics. Journal of the European Ceramic Society, 2017, 37(13): 4059-4066.

[11] SHANG SHAN SHAN, HE XIONG, YANG YI, et al. Preparation of $\mathrm{Ti}_{2} \mathrm{AlN}$ porous ceramics by organic foam impregnation process. Key Engineering Materials, 2016, 697: 163-168.

[12] QIAN HAO-RAN, CHENG XU-DONG, ZHANG HE-PING, et al. Preparation of porous mulliteceramics using fly ashcenosphere as a pore-forming agent by gelcastingprocess. International Journal of Applied Ceramic Technology, 2014, 11(5): 858-863.

[13] GE SHENG-TAO, LIN LIANG-XU, ZHANG HAI-JUN, et al. Synthesis of hierarchically porous mullite ceramics with improved thermal insulation via foam-gelcasting combined with pore former addition. Advances in Applied Ceramics, 2018, 117(8):493-499.

[14] CHAU TRANG THE LIEU, LE DUNG QUANG TIEN, LE HOA THI, et al. Chitin liquid-crystal-templated oxide semiconductor aerogels. ACS Applied Materials \& Interfaces, 2017, 9(36): 30812-30820.

[15] JESÚS M RODRÍGUEZ-PARRA, RODRIGO MORENO, MARÍA ISABEL NIETO. Effect of cooling rate on the microstructure and porosity of alumina produced by freeze casting. Serbian Chemical Society Journal, 2012, 77(12): 1775-1785.

[16] DU JIAN-CONG, ZHANG XING-HONG, HONG CHANG-QING, et al. Microstructure and mechanical properties of $\mathrm{ZrB}_{2}-\mathrm{SiC}$ porous ceramic by camphene-based freeze casting. Ceramics International, 2013, 39(2): 953-957.

[17] XIA YONG-FENG, ZENG YU-PING, JIANG DONG-LIANG. Microstructure and mechanical properties of porous $\mathrm{Si}_{3} \mathrm{~N}_{4}$ ceramics prepared by freeze-casting. Materials \& Design, 2012, 33: 98-103.

[18] REN LIN-LIN ZENG YU-PING JIANG DONG-LIANG. Preparation of porous $\mathrm{TiO}_{2}$ by a novel freeze casting. Ceramics International, 2009, 35(3): 1267-1270. 
[19] BI YU-BAO, HAN LEI, ZHENG YANG-FAN, et al. Lotusseedpod-bioinspired 3D superhydrophobic diatomite porous ceramics comodified by graphene and carbon nanobelts. ACS Applied Materials \& Interfaces, 2018, 10(32): 27416-27423.

[20] MA YING, ZHANG GAO-KE. Sepiolite nanofiber-supported platinum nanoparticle catalysts toward the catalytic oxidation of formaldehyde at ambient temperature: efficient and stable performance and mechanism. Applied Clay Science, 2016, 288: 70-78.

[21] SUÁREZ M, GARCÍA-ROMERO E. Variability of the surface properties of sepiolite. Applied Clay Science, 2012, 67: 72-82.

[22] LI TIAN, WANG LI-JUAN, WANG KAI-LEI, et al. The preparation and properties of porous sepiolite ceramics. Scientific Reports, 2019, 9(1): 7337.

[23] VICTORIA BERNARDO, FREDERIK VAN LOOCK, JUDITH MARTIN-DE LEON, et al. Mechanical properties of PMMAsepiolite nanocellular materials with a bimodal cellular structure. Macromolecular Materials and Engineering, 2019, 304(7): 1900041.

[24] GUO ZHEN-HUA, LIU ZHONG-TAO, YANG FAN, et al. Application of sepiolite in wastewater treatment. Environmental Protection and Circular Economy, 2016, 36(10): 30-32.

[25] MILT V G, BANUS E D, MIRÓ E E, et al. Structured catalysts containing $\mathrm{Co}, \mathrm{Ba}$ and $\mathrm{K}$ supported on modified natural sepiolite for the abatement of diesel exhaust pollutants. Chemical Engineering Journal, 2010, 157(2/3): 530-538.

[26] WANG JUN-KAI, DENG XIAN-GONG, ZHANG HAI-JUN, et al. Synthesis of carbon nanotubes via Fe-catalyzed pyrolysis of phenolic resin. Physica E, 2017, 86: 24-35.

[27] WANG JUN-KAI, DENG XIAN-GONG, ZHANG HAI-JUN, et al. Carbon nanotube preparation by catalytic pyrolysis of phenolic resin with nickel nitrate. Materials for Mechanical Engineering, 2016, 40(8): 30-33.

[28] SONG JIAN-BO, ZHANG HAI-JUN, WANG JUN-KAI, et al. High-yield production of large aspect ratio carbon nanotubes via catalytic pyrolysis of cheap coal tar pitch. Carbon, 2018, 130: 701-713.

[29] RAJAKOVIĆ-OGNJANOVIĆ V, ALEKSIĆ G, RAJAKOVIĆ L. Governing factors for motor oil removal from water with different sorption materials. Journal of Hazardous Materials, 2008, 154(1/2/3): 558-563.

[30] AKHAVAN B, JARVIS K, MAJEWSKI P. Hydrophobic plasma polymer coated silica particles for petroleum hydrocarbon removal.
ACS Applied Materials \& Interfaces, 2013, 5(17): 8563-8571.

[31] YUAN WEI-WEI, YUAN PENG, LIU DONG, et al. A hierarchically porous diatomite/silicalite-1 composite for benzene adsorption/desorption fabricated via a facile pre-modification in situ synthesis route. Chemical Engineering Journal, 2016, 294: 333-342.

[32] XUE YANG-MING, DAI PENG-CHENG, JIANG XIANG-FEN, et al. Template-free synthesis of boron nitride foam-like porous monoliths and their high-end applications in water purification. Journal of Materials Chemistry A, 2016, 4(4): 1469-1478.

[33] DONG BING-BING, YANG MING-YE, WANG FEI-HONG, et al. Porous $\mathrm{Al}_{2} \mathrm{O}_{3}$ plates prepared by combing foaming and gel-tape casting methods for efficient collection of oil from water. Chemical Engineering Journal, 2019, 370: 658-665.

[34] HAO WEN-TAO, XU JIAN, LI RAN, et al. Developing superhydrophobic rock wool for high-viscosity oil/water separation. Chemical Engineering Journal, 2019, 368: 837-846.

[35] GEN HAYASE, KAZUYOSHI KANAMORI, MASASHI FUKUCHI, et al. Facile synthesis of marshmallow-like macroporous gels usable under harsh conditions for the separation of oil and water. Angewandte Chemie International Edition, 2013, 52(7): 1986-1989.

[36] ZHANG AI-LI, ZHAI XIU-JING, FU YAN, et al. Reach on electrochemical lithium storage performance of carbon nanotubes. Journal of Inorganic Materials, 2004, 19(1): 244-248.

[37] ZHENG YANG-FAN, ZHANG HAI-JUN, GE SHENG-TAO, et al. Synthesis of carbon nanotube arrays with high aspect ratio via $\mathrm{Ni}$-catalyzed pyrolysis of waste polyethylene. Nanomaterials, 2018, 8(7): 556.

[38] WENZEL ROBERT N. Resistance of solid surfaces to wetting by water. Industrial \& Engineering Chemistry, 1936, 28(8): 988-994.

[39] CAO SU-JIAO, QIU FANG, XIONG CHEN, et al. Superhydrophobic PES/PDA/ODTS fibrous mat prepared by electrospinning and silanization modification for oil/water separation. Journal of Applied Polymer Science, 2018, 135(12): 45923.

[40] CHENG PENG, JING PENG, ZHANG SHI-YUAN, et al. A macroporous metal-organic framework with enhanced hydrophobicity for efficient oil adsorption. Chemistry-A European Journal, 2018, 24(15): 3754-3759.

[41] GUPTA SHIVAM, TAI NYAN-HWA. Carbon materials as oil sorbents: a review on the synthesis and performance. Journal of Materials Chemistry A, 2016, 4(5): 1550-1565. 